\title{
A Challenging to Characterization of Superconductors: Accurate Measurements of Charge Distribution and Interfacial Displacement
}

\author{
Yimei Zhu,* Lijun $\mathrm{Wu}^{*}$ and J. Tafto** \\ *Material Science Division, Brookhaven National Laboratory, Upton, NY 11973, USA \\ **also Department of Physics, University of Oslo, P.O. Box 1048 Blindern, 0316 Oslo, Norway
}

One of the most challenging tasks in solid-state physics and materials science is to understand electronic properties of crystals and interfaces. This is particular important for superconductors where electron/hole distribution in crystal and grain boundary determines the usefulness of the materials. To meet the challenge, we developed a novel electron-diffraction technique PARODI (Parallel Recording Of Dark-field Images) by focusing a small probe above the sample to measure charge density and lattice displacement. Since the method couples diffraction with images, it is thus suitable for studying crystals as well as defects. Combined with HREM and ELNES, the method allows us to address issues crucial to superconductivity mechanism.

To measure 1-D charge distribution, we record PARODI patterns of a systematic reflection row from a wedge crystal. Through quantitative intensity analysis, i.e., comparing the experiments with calculations via fitting and error analyses, we can accurately determine the structure factors of loworder reflections of crystals with a large unit cell, and thus valence electron distribution and charge transfer with very high accuracy [1]. In the case of $\mathrm{YBa}_{2} \mathrm{Cu}_{3} \mathrm{O}_{7}$, a movement of 0.05 electron between $\mathrm{CuO}$ and $\mathrm{CuO}_{2}$ planes that corresponds to rearranging 1 out of 5000 electrons in the crystal, changes the 001 structure factor of electron diffraction by $1 \AA$ while we determine this structure factor with an accuracy of $0.5 \AA$. Fig. 1 shows the charge-density distribution $\rho$, along the c-axis for $\mathrm{Bi}_{2} \mathrm{Sr}_{2} \mathrm{CaCu}_{2} \mathrm{O}_{8}(\mathrm{Bi} / 2212)$ together with the charge transfer $\Delta \rho$, determined from the measurement and the first principle calculation, using formal valence as a reference. A 2-D charge density map of $\mathrm{MgB}_{2}$ projected along the [001] direction is shown in Fig.2. It reveals not only bonding characteristics ( $\mathrm{B}$ : covalent bonding, $\mathrm{Mg}$ : close to ionic bonding) but also significant charge transfer from $\mathrm{Mg}$ to $\mathrm{B}$. Measurements of electrostatic potential variation across grain boundaries in $\mathrm{Bi} / 2212$ using PARODI and electron holography will also be presented.

A remarkable advantage of PARIDO is to simultaneously study charge distribution and lattice displacement from the same illuminated area because of a wide range of reflections available (electrons scattered at small-angles are sensitive to charge arrangement, while those scattered at large-angles are sensitive to crystal distortion). Fig.3 is a calculated PARODI pattern with a stacking fault in the middle. The interfacial interference fringes are out of contrast in reflections where $\mathbf{g} \cdot \mathbf{R}$ equals to an integer. We have measured grain boundary expansion of several (001) twist boundaries in $\mathrm{Bi} / 2212$. A typical HREM image of such a boundary [2] is shown in Fig.4. By examining the fringe contrast in very high-order reflections such as 0030 , we were able to determine the interfacial lattice displacement with an unprecedented accuracy down to 1pm [1]. The displacement was found to range from 0.2-0.4 $\AA$ which was not detectable using HREM. An example on the study of a $5^{\circ}$ tilt boundary in Bi/2212 using PARODI is presented in Fig.5. [3]

\section{References}

[1] Wu, Zhu and Tafto, Phys.Rev.B, 59, 6035 (1999), Phys. Rev. Lett. 855126 (2000).

[2] Y.Zhu, Q.Li, Y.N.Tsay, M.Suenaga, G.D.Gu, and N.Koshizuka, Phys.Rev.B, 57, 8601 (1998).

[3] Work supported by US DOE under contract No. DE-AC02-98CH10886. 


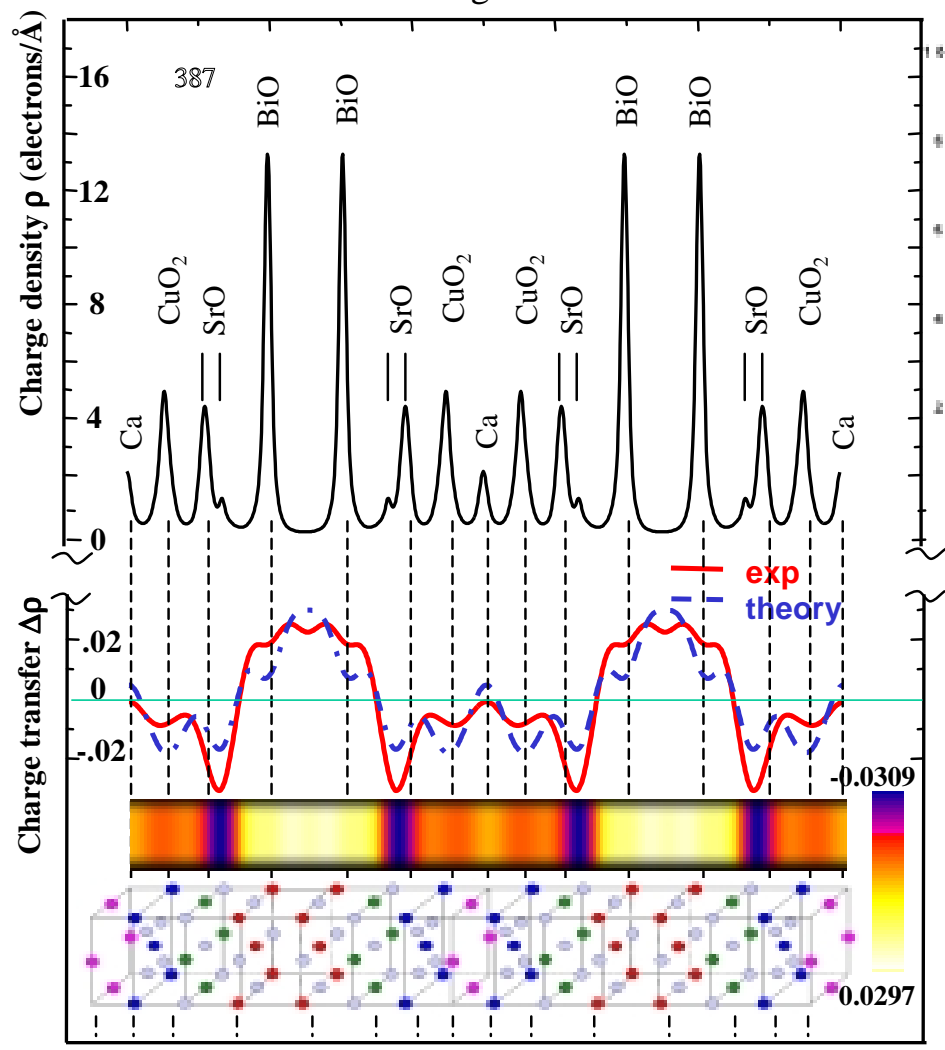

Fig.2

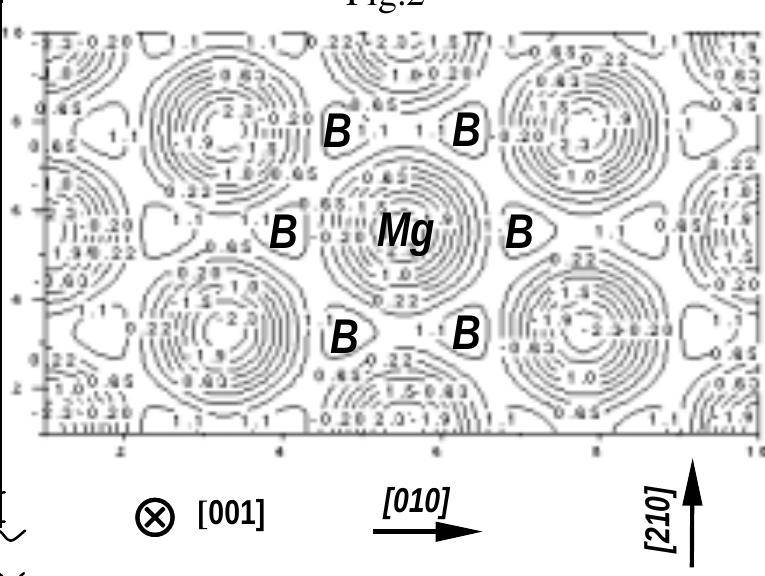

Fig.2 The projected two-dimensional valence-electron distribution map of $\mathrm{MgB}_{2}$ along the [001] direction using neutral atoms as a reference.

Fig1 Charge-density distribution, $\rho$, along the caxis for $\mathrm{Bi} / 2212$ together with the charge transfer, $\Delta \rho$, determined from the experimental measurement and the theory (Gupta model).

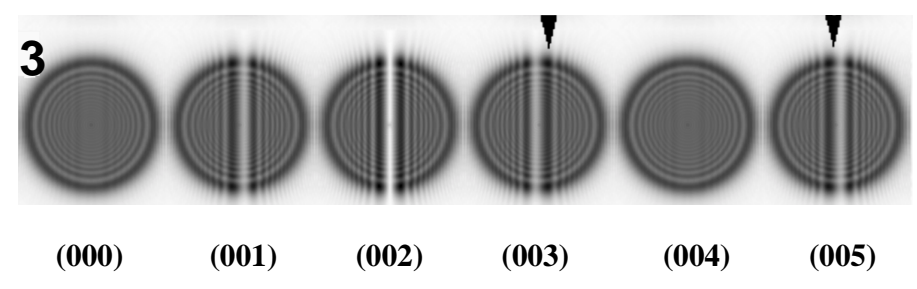

Fig.3 Calculated interferometric PARODI pattern involving a stacking fault with a displacement $\mathrm{R}=1 / 4 c$. The asymmetry of the interfacial fringes (marked by arrows) can be used to determine the sign of the R.

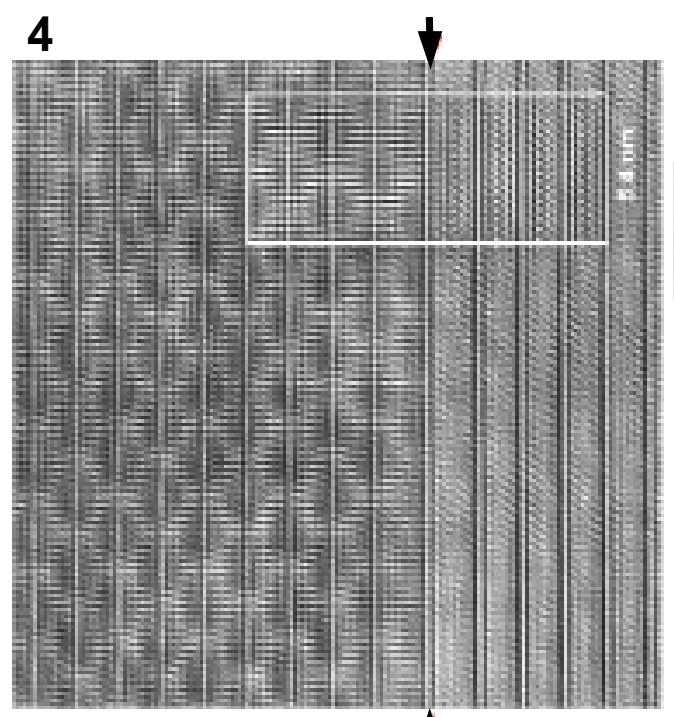

Fig.4 High resolution image of a (001) twist boundary in Bi/2212. The embedded image is a simulated one incorporated with charge.

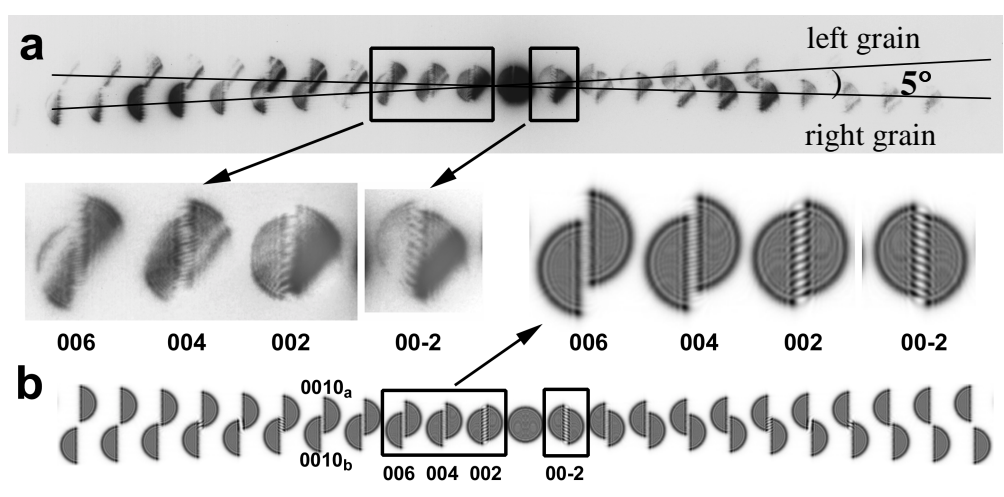

Fig.5 Experimental (a) and calculated (b) PARODI patterns of a $5^{\circ}$

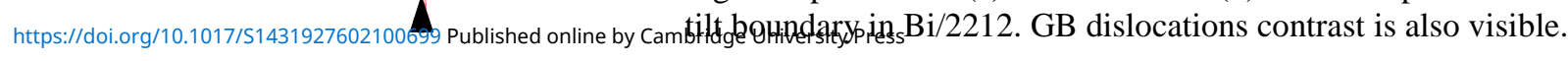

